\title{
Postpartum sexual function; the importance of the levator ani muscle
}

\author{
Anne-Marie $\operatorname{Roos}^{1}$ (D) $\cdot$ Leonie Speksnijder ${ }^{2} \cdot$ Anneke B. Steensma $^{1}$ \\ Received: 12 October 2019 / Accepted: 29 January 2020 / Published online: 24 February 2020 \\ (C) The Author(s) 2020
}

\begin{abstract}
Introduction and hypothesis Pelvic floor muscle function plays an important role in female sexual functioning. Smaller genital hiatal dimensions have been associated with sexual dysfunction, mainly dyspareunia. On the other hand, trauma of the levator ani muscle sustained during childbirth is associated with increased genital hiatus, which potentially can affect sexual functioning by causing vaginal laxity. This study aims to determine the association between levator hiatal dimensions and female sexual dysfunction after first vaginal delivery.

Methods This is a secondary analysis of a prospective observational study. Two hundred four women who had a first, spontaneous vaginal delivery at term between 2012 and 2015 were recruited at a minimum of 6 months postpartum. Thirteen pregnant women were excluded. We analyzed the association of total PISQ-12 score, as well as individual sexual complaints (desire, arousal, orgasm and dyspareunia), with levator hiatal dimensions at rest, with maximum Valsalva and during pelvic floor muscle contraction as measured by 4D transperineal ultrasound. Statistical analysis was performed using linear regression analysis and Mann-Whitney U test.

Results One hundred ninety-one women were evaluated at a median of 11 months postpartum. There was no significant association between total PISQ-12 score and levator hiatal dimensions. Looking at individual sexual complaints, women with dyspareunia had significantly smaller levator hiatal area and anterior-posterior diameter on maximum Valsalva. By using multivariate logistic regression analysis however we found dyspareunia was not independently associated with levator hiatal dimensions.
\end{abstract}

Conclusions After first vaginal delivery sexual dysfunction is not associated with levator hiatal dimensions as measured by 4D transperineal ultrasound.

Keywords Levator ani muscle · Pelvic floor · Postpartum · Sexual dysfunction · Ultrasonography

\section{Introduction}

Childbirth can have a great impact on women's sexual functioning. Postpartum sexual functioning is influenced by both psychological changes associated with the transition into parenthood as well as physical changes such as perineal trauma [1]. Female sexual dysfunction in the DSM V is subdivided into three broad categories,

Anne-Marie Roos

annemarie.roos@gmail.com

1 Department of Obstetrics and Gynecology, Division of Urogynaecology, Erasmus Medical Centre, Doctor Molewaterplein 40, 3015, GD Rotterdam, The Netherlands

2 Department of Obstetrics and Gynecology, division of Urogynaecology, Amphia Hospital, Breda, The Netherlands including: sexual interest/arousal disorder, female orgasmic disorder and genitopelvic pain/penetration disorder, including dyspareunia [2]. Previous literature on postpartum sexual function has focused mainly on the complaint of dyspareunia, which is associated with instrumental delivery and the extent of perineal trauma sustained [3].

Physical birth trauma encompasses more than perineal trauma alone. Following first vaginal delivery $13-36 \%$ of women sustain trauma of the levator ani muscle [4-7]. This trauma can be divided into levator ani muscle avulsion and levator over-distension or a combination of both. Trauma of the levator ani muscle is associated with increased genital hiatus (levator ballooning) and decreased pelvic floor contractility and is strongly associated with symptoms and clinical signs of prolapse [4, 6-9]. This potentially could affect sexual functioning, as decreased pelvic floor muscle strength has been associated with worse sexual function [10-12]. 
Literature on the association of genital hiatus, levator trauma and postpartum sexual functioning is sparse. At short-term follow-up levator avulsion was associated with symptoms of vaginal laxity and reduced vaginal sensation, but these complaints did not have appreciable effects on women's sexual functioning $[13,14]$. At 1 year postpartum women with persistent levator avulsion had significantly more bothersome symptoms of a loose vagina. A trend towards a spoiled sex life as a result of these symptoms was found, but did not reach significance [15].

On the other hand, smaller hiatal dimensions have been associated with sexual dysfunction as well, mainly dyspareunia. Women with provoked vestibulodynia (PVD) were shown to have smaller relative increases in hiatal area on maximum Valsalva, which is associated with hypertonia from the pelvic floor muscles [16].

The aim of this study was to determine the nature of pelvic floor muscle involvement in women with sexual dysfunction after first vaginal delivery. We hypothesized that larger levator hiatus and decreased contractibility of the levator ani muscle are associated with female sexual dysfunction, because of the association with symptoms of reduced vaginal sensation and vaginal laxity. We also hypothesized that a small hiatus in rest and decreased relaxation are associated with dyspareunia. For this purpose we evaluated the biometry of the levator hiatus at rest, pelvic floor muscle contraction (PFMC) and maximal Valsalva using $3 \mathrm{D} / 4 \mathrm{D}$ transperineal ultrasound in relation to sexual complaints.

\section{Materials and methods}

This is a secondary analysis of a prospective observational study which aimed to evaluate the association of mediolateral episiotomy with levator injury and urogynecological complaints (EpiLeva study) [17]. All participants were recruited at the Amphia Hospital in Breda, The Netherlands, between 2012 and 2015. Women who underwent a first, spontaneous, term vaginal delivery of a singleton in cephalic position were included. Women who delivered prematurely, who delivered a fetus in non-cephalic position, who experienced instrumental vaginal delivery, obstetric anal sphincter injury or cesarean section were not included. The investigators were not involved in the management of the participants' deliveries.

Women who agreed to participate filled out standardized urogynecological questionnaires, and a detailed examination including 3D/4D transperineal ultrasound was performed at a minimum of 6 months postpartum [17]. Antenatal and intrapartum characteristics were retrospectively obtained from the patients' medical notes.

\section{Assessment of sexual function}

For assessment of sexual function the Dutch validated version of the Pelvic Organ Prolapse/Urinary Incontinence Sexual Questionnaire-12 (PISQ-12) was used [18]. The PISQ-12 is a 12 -item, condition-specific tool for assessing sexual function in women with urinary incontinence and/or pelvic organ prolapse. Responses are measured on a 5-point Likert scale ranging from "never" to "always." The total score ranges from 0 to 48 , with higher scores indicating better sexual function. For analysis of sexual function in this study we analyzed the total PISQ score as well as individual sexual complaints. To define individual complaints we dichotomized PISQ questions. Dyspareunia was defined as answering "always" or "usually" to the question: "Do you feel pain during sexual intercourse?" Reduced desire was defined as answering "seldom" or "never" to the question: "How often do you feel sexual desire?" Inability to reach an orgasm was defined as answering "seldom" or "never" to the question: "Do you climax (have an orgasm) while having sexual intercourse with your partner?" Reduced arousal was defined as answering "seldom" or "never" to the question: "Do you feel sexually excited (turned on) when having sexual activity with your partner?"

\section{Three/four-dimensional transperineal ultrasound}

Three/four-dimensional transperineal ultrasound was performed in supine position and after voiding, using a Voluson E Expert system using a 4-8-MHz RAB abdominal probe (GE Healthcare, Chalfont St. Giles, UK) as previously described by Dietz et al. [19]. The ultrasound volumes were obtained at rest, PFMC and maximal Valsalva. All volumes were obtained by an investigator experienced in performing 3D/4D transperineal ultrasound.

Offline analysis of the recorded 3D/4D transperineal ultrasound volumes were performed by experienced investigators who were blinded against all clinical and obstetric data [17]. Analysis was done using specialized 3D imaging software, 4D View version 17.0 (GE Healthcare, Chalfont St. Giles, UK). The anteriorposterior (AP) diameter of the levator hiatus, left-right (LR) transverse diameter of the levator hiatus and area of the levator hiatus were measured at rest, PFMC and maximal Valsalva (Fig. 1). Relative changes in levator hiatal dimensions between rest and maximum Valsalva ([dimension at maximum Valsalva - dimension at rest/ dimension at rest] $\times 100 \%$ ) and between rest and PFMC ([dimension at contraction - dimension at rest/dimension at rest] $\times 100 \%)$ were computed [16]. 


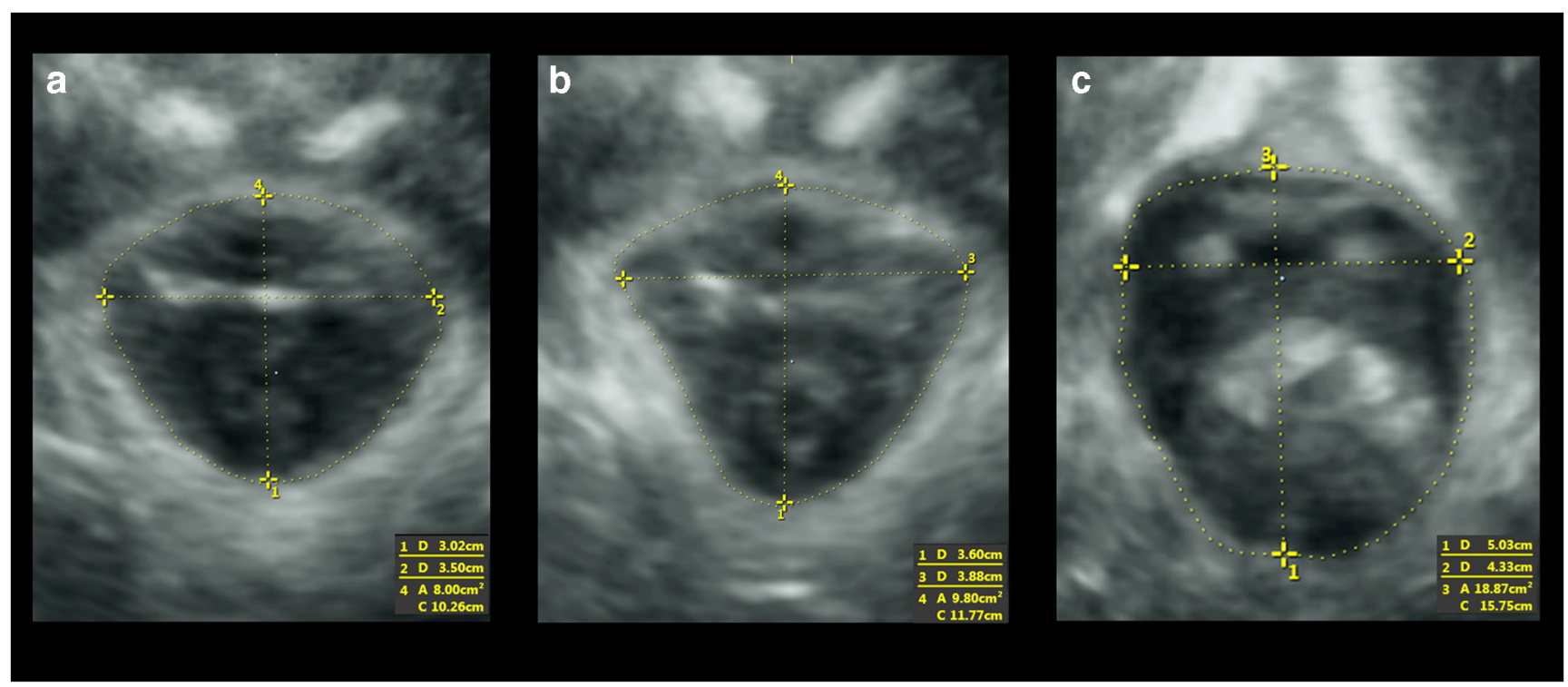

Fig. 1 Example of measurement of levator hiatal dimensions in one patient at pelvic floor muscle contraction, at rest and maximal Valsalva

\section{Statistical analysis}

For this analysis we excluded women who were pregnant at the time of examination, as pregnancy has the potential to compromise sexual function and sexual activity [20,21].

Measurements of hiatal dimensions followed a nonnormal distribution. Linear regression analysis was used to compare measurements of hiatal dimensions and total PISQ-12 score. We used $\mathrm{R}^{2}$ to interpret the explained variance. Mann-Whitney $U$ test was used to compare hiatal dimensions between women with and without individual sexual complaints. Baseline and obstetric factors were compared between women with and without sexual complaints using Student's t-test for continuous variables, Kendall's tau-c for ordinal variables and chisquare/fisher's exact test for categorical variables. Significant univariate associations were subjected to multivariate analysis using logistic regression analysis.

As this was a secondary analysis of the EpiLeva data, the sample size was determined by the primary end points of the EpiLeva study [17] and not specific to the research question addressed in this article. Statistical analysis was performed using IBM SPSS Statistics version $25 . P$ values $<0.05$ were considered statistically significant.

\section{Ethical approval}

Ethical approval for this study was obtained from the medical research ethics committee of the Erasmus Medical Centre (MEC-2012-058). Date of approval was 11 April 2012.

\section{Results}

A total of 2535 women were invited, and 204 (8\%) agreed to participate in our study. Comparison of baseline characteristics between participants and nonparticipants was previously described [17]. In summary, participants were more likely to be Caucasian and had a longer second stage of labor. In non-participants more women were induced and more frequently received epidural analgesia. There were no significant differences in age, BMI, episiotomy rate and other obstetrical factors.

Thirteen $(6 \%)$ women were pregnant at the time of examination and were excluded from the analysis. The median time at investigation after vaginal delivery was 11 months (range 6-33). The baseline and obstetric characteristics of the 191 included women are shown in Table 1.

Table 1 Baseline and obstetric characteristics of 191 included women in $\mathrm{N}(\%)$

Baseline

Age

$30(20-40) *$

Ethnicity: Caucasian

$174(91 \%)$

BMI at booking

$24(16-39)^{*}$

Obstetric

Perineal tear

Intact perineum

$39(21 \%)$

1 st degree tear

$16(8 \%)$

2nd degree tear

$38(20 \%)$

Episiotomy

$98(51 \%)$

\footnotetext{
*Mean (range)
} 


\section{Sexual functioning and levator hiatal dimensions}

All women had resumed sexual activity at the time of examination. Median (range) of the PISQ-12 was 38 (22-46). In our population 31 (16\%) women experienced dyspareunia, 24 $(13 \%)$ had difficulty reaching an orgasm, $33(17 \%)$ experienced reduced desire, and $7(4 \%)$ had a problem with arousal.

Using linear regression analysis we did not find a significant association between total PISQ-12 score and (relative changes in) levator hiatal dimensions, accept for AP diameter at rest $(p=0.014)$. Larger diameter is associated with higher PISQ-12 score and thus with better sexual functioning. However, this association is very weak, as $<5 \%$ of variance in the PISQ score is explained by the AP diameter at rest $\left(\mathrm{R}^{2}=0.031\right)$.

Table 2 shows the comparison of hiatal dimensions between women with and without dyspareunia. These results indicate that women with dyspareunia had significantly smaller levator hiatal areas and AP diameter on maximum Valsalva. No significant effects were found for LR diameter. Table 3 shows the comparison in relative changes between levator hiatal dimensions between rest and PFMC and between rest and maximal Valsalva in women with and without dyspareunia. This showed no significant differences.

For the other individual sexual complaints there was no significant difference between levator hiatal dimensions and relative changes in levator hiatal dimensions. There were no significant differences between women with and without reduced desire, women with and without reduced arousal and women with and without difficulty reaching an orgasm.

\section{Multivariate analysis}

We used multivariate analysis to correct for age, BMI at booking, ethnicity (Caucasian vs. non-Caucasian), postnatal follow-up time (weeks), grade of tear/episiotomy and other sexual complaints including reduced desire, reduced arousal and difficulty reaching an orgasm. Variables that showed a (near) significant association at univariate analysis were included in a multivariate model. In the final model for dyspareunia we included age, ethnicity, hiatus at maximal Valsalva, AP diameter at maximal Valsalva, the presence of reduced desire and reduced arousal. On multivariate analysis using logistic regression we found dyspareunia was independently significantly associated with younger age, reduced desire and reduced arousal, but not with hiatal dimensions at maximal Valsalva.

\section{Discussion}

This study aimed to determine the association between levator hiatal dimensions and relative changes in levator hiatal dimensions and sexual dysfunction after first vaginal delivery. All women were sexually active at time of investigation. Sixteen percent of women experienced dyspareunia, $13 \%$ had difficulty reaching an orgasm, $17 \%$ experienced reduced desire, and $4 \%$ had a problem with arousal. Postpartum sexual dysfunction (including dyspareunia) is identified in $41-83 \%$ of women at $2-$ 3 months postpartum [1]. Longer term data beyond 6 months postpartum are sparse. There is great variability in the literature on the reported prevalence rates of particular sexual dysfunctions due to differences in the definition of sexual dysfunction, degree of severity and differences in age groups. Similar to our data, prevalence rates for women in the general population vary from $17 \%$ to $55 \%$ for low levels of sexual desire, from $8 \%$ to $28 \%$ for arousal and lubrication problems, from $16 \%$ to $25 \%$ for orgasmic dysfunction and from $14 \%$ to $27 \%$ for dyspareunia [22].
Table 2 Median (range) levator hiatal dimensions at rest, on pelvic floor muscle contraction and on maximal Valsalva in women with and without dyspareunia using Mann-Whitney U test

\begin{tabular}{lccc}
\hline & $\begin{array}{l}\text { Dyspareunia } \\
N=31\end{array}$ & $\begin{array}{l}\text { No dyspareunia } \\
N=160\end{array}$ & \\
& & & \\
\hline Rest value \\
Hiatal area & $13.12(7.84-20.53)$ & $14.03(8.18-34.13)$ & 0.133 \\
AP diameter $(\mathrm{cm})$ & $4.92(3.01-6.06)$ & $5.09(3.67-7.22)$ & 0.074 \\
LR diameter $(\mathrm{cm})$ & $3.77(3.09-5.80)$ & $3.92(2.66-6.09)$ & 0.402 \\
Contraction & $10.72(7.13-19.39)$ & & \\
Hiatal area & $4.21(2.87-5.54)$ & $4.81(6.68-26.28)$ & 0.149 \\
AP diameter & $3.73(2.64-5.46)$ & $3.62(2.63-6.17)$ & 0.328 \\
LR diameter & $16.13(7.90-33.43)$ & $18.79(7.38-53.69)$ & 0.450 \\
Valsalva & $5.25(3.22-6.95)$ & $5.86(3.18-28.91)$ & 0.035 \\
Hiatal area & $4.26(2.83-6.78)$ & $4.23(2.94-7.41)$ & 0.006 \\
AP diameter & & & 0.467 \\
LR diameter & &
\end{tabular}


Table 3 Relative changes [median (range)] in levator hiatal dimensions between rest and pelvic floor muscle contraction and between rest and maximal Valsalva in women with and without dyspareunia using MannWhitney U test

\begin{tabular}{llll}
\hline & $\begin{array}{l}\text { Dyspareunia } \\
\mathrm{N}=31\end{array}$ & $\begin{array}{l}\text { No dyspareunia } \\
\mathrm{N}=160\end{array}$ & $P$ value \\
\hline Contraction & & & \\
Hiatal area (\%) & $-16.05(-44.22-5.55)$ & $-18.63(-55.18-69.49)$ & 0.386 \\
AP diameter (\%) & $-13.69(-32.54-11.25)$ & $-15.78(-44.12-45.66)$ & 0.236 \\
LR diameter (\%) & $-4.58(-22.00-22.12)$ & $-7.73(-32.68-28.45)$ & 0.149 \\
Valsalva & & & \\
Hiatal area (\%) & $30.60(-28.74-91.80)$ & $31.87(-29.78-197.87)$ & 0.308 \\
AP diameter $(\%)$ & $10.25(-19.04-61.79)$ & $15.35(-21.87-456.32)$ & 0.230 \\
LR diameter $(\%)$ & $7.03(-28.54-53.57)$ & $9.43(-21.66-63.29)$ & 0.479 \\
\hline
\end{tabular}

We hypothesized that women that experience sexual complaints show significant differences in levator hiatal dimensions and relative changes in these dimensions on PMFC and maximal Valsalva compared with women without sexual complaints. However, our results showed that 11 months after first vaginal delivery hiatal dimensions are not predictive of overall sexual dysfunction as measured by PISQ-12. When we studied individual sexual complaints we found that dyspareunia was associated with smaller hiatal dimensions at Valsalva on univariate analysis, but on multivariate analysis, hiatal dimensions were not an independent risk factor for dyspareunia.

Previous studies showed that at 5 months after delivery, levator ani muscle avulsion and levator over-distension did not have appreciable effects on women's sexual functioning related to sexual activity, sensation on intercourse, arousal and orgasm [14]. Although women with persistent levator avulsion had significantly more bothersome symptoms of a loose vagina at 1 year postpartum, these symptoms had no impact on their sexual life [15]. Although levator avulsion was associated with larger hiatal areas on transperineal ultrasound in these studies, the association between the different levator hiatal dimensions and sexual function was not examined. A study by Aydin et al. did correlate levator hiatal dimensions and sexual dysfunction in non-symptomatic sexually active premenopausal women [23]. An increase in AP diameter of the levator hiatus during maximal Valsalva was weakly associated with worse sexual functions, particularly desire, arousal and orgasm domains [23]. Our study demonstrates that after first vaginal delivery hiatal dimensions were not associated with arousal, desire or orgasm. Although not significant on multivariate analysis, we did find that women with dyspareunia had smaller hiatal dimensions at Valsalva rather than larger dimensions, which could be a sign of higher pelvic floor muscle tone.

The etiology of sexual dysfunction is multifactorial, including emotional, physical and psychological causes. Changes in the dimensions and contractility of the pelvic floor might be the main cause of sexual dysfunction only in a subgroup of women. Perineal pain resulting from perineal trauma, fear of pain on resumption of intercourse or psychological stress associated with changes in family structure following childbirth could possibly cause an increase in pelvic floor muscle tone in the postpartum period. Previous studies have shown that levator hiatal dimensions are smaller in women diagnosed with provoked vestibulodynia (PVD). In a study by Thibault et al., women were diagnosed with PVD when complaining about pain around the vaginal opening upon attempted vaginal penetration for a minimum of 6 months [16]. The diagnosis was confirmed by using a standardized cotton-swab test. Women with PVD had smaller hiatal dimensions at rest, on PFMC and on maximum Valsalva than controls. Furthermore, these women had smaller relative increases in hiatal area on maximum Valsalva, which is associated with hypertonia from the pelvic floor muscles.

When dyspareunia is caused by, or maintained as a result of, pelvic floor hypertonia without anatomical causes, firstline treatment consists of education, tactile desensitization and pelvic floor physiotherapy possibly in combination with psychological or sexological consultation. When first-line treatment fails, more invasive interventions can be considered. One of these invasive interventions is injection with botulinum toxin A (BTA) in the pelvic floor muscles. When injected intramuscularly BTA produces a localized, partial and reversible chemical denervation of the muscle, which results in localized muscle weakness or paralysis. There is some lowgrade evidence that injection of BTA in the hypertonic pelvic floor muscles decreases pelvic floor resting pressure as measured by manometry and improves dyspareunia in women with therapy-resistant chronic pelvic pain [24, 25]. Pelvic floor ultrasound might play a role in the future in the diagnostic workup of women with dyspareunia. A cutoff value for high muscle tone needs to be determined. These women with high muscle tone could be a subgroup of women with dyspareunia that might benefit from BTA injections.

To the best of our knowledge, this is the first study to evaluate the association of sexual complaints and the biometry of the levator hiatus and relative changes of the puborectalis muscle using 3D/4D transperineal ultrasound in the postpartum period. All women in our study filled out the validated PISQ-12 questionnaire. The women included in our study did 
not have an increased risk for sexual dysfunction postpartum, as women who had instrumental delivery or third- or forth degree perineal tearing were excluded [1]. Furthermore, the researchers who conducted the study were blinded against all clinical and obstetric data during analysis of the ultrasound data, thereby reducing bias.

There were also some limitations to our study. First, there was a possibility of selection bias. Only $8 \%$ of invited women participated in our study. Lack of time and fear of the examination were the most mentioned reasons for non-participation. This might have led to a higher participation rate of women with complaints or complications. However, the percentage of women reporting sexual complaints in our study does not exceed the incidence reported in previous literature, suggesting a representative cohort [22]. Participants were more likely to be Caucasian, but did not differ in age and BMI compared with non-participants. We therefore believe that our results can be generalized to other populations of mainly Caucasian women who had a first spontaneous vaginal delivery at term. A second limitation is our sample size. As this was a secondary analysis of the EpiLeva data, the sample size was determined by the primary end points of the EpiLeva study and not specific to the research question addressed in this article [17]. It might be that our sample size was too small to evaluate a significant association between the biometry of the levator hiatus and sexual complaints. A third limitation is the fact that we did not ask about sexual functioning before and during pregnancy. However, due to the design of our study, it was impossible to prospectively collect these data as it would have required inclusion of study participants at the first antenatal booking. When information on pre-pregnancy sexual functioning was obtained retrospectively in the postpartum period, it might have been subject to recall bias. Finally, the PISQ-12 questionnaire may not be the best questionnaire to identify sexual dysfunction in our cohort of postpartum women. The PISQ-12 is validated to assess sexual function in women with urinary incontinence and/or pelvic organ prolapse, but has previously also been used to evaluate sexual function after vaginal delivery [26, 27]. The EpiLeva study was conducted to assess the association of mediolateral episiotomy with levator injury and urogynecological complaints. This is why we chose to use the PISQ-12 questionnaire. However, the frequency of pelvic organ prolapse and urinary incontinence symptoms in our study population was low. As the PISQ-12 does not provide cutoff values discriminating between normal sexual function and sexual dysfunction, we dichotomized answers of questions to what we considered to be relevant complaints. For future research on this topic a more general female sexual function questionnaire could be considered, for example, the Female Sexual Function Index (FSFI).

In conclusion, we can state that sexual dysfunction at 11 months postpartum is not independently associated with levator hiatal dimensions or relative changes in levator hiatal dimensions as measured by 3D/4D transperineal ultrasound. Although women with dyspareunia had smaller hiatal dimensions at maximum Valsalva, suggesting higher pelvic floor muscle tone, on multivariate analysis this association was not significant. Sexual dysfunction in the postpartum period is multifactorial and can have many different psychological as well as physical causes. Changes in hiatal dimensions of the pelvic floor could be one of the causes of sexual dysfunction in a certain group, but further research is necessary to identify these women.

Author participation AM Roos: Data analysis, Manuscript writing/ editing.

L Speksneijder: Protocol/project development, Data collection, Data analysis, Manuscript writing/editing.

AB Steensma: Protocol/project development, Data analysis, Manuscript writing/editing.

\section{Compliance with ethical standards}

Conflict of interest The authors declare that they have no conflict of interest.

Open Access This article is licensed under a Creative Commons Attribution 4.0 International License, which permits use, sharing, adaptation, distribution and reproduction in any medium or format, as long as you give appropriate credit to the original author(s) and the source, provide a link to the Creative Commons licence, and indicate if changes were made. The images or other third party material in this article are included in the article's Creative Commons licence, unless indicated otherwise in a credit line to the material. If material is not included in the article's Creative Commons licence and your intended use is not permitted by statutory regulation or exceeds the permitted use, you will need to obtain permission directly from the copyright holder. To view a copy of this licence, visit http://creativecommons.org/licenses/by/4.0/.

\section{References}

1. Leeman LM, Rogers RG. Sex after childbirth: postpartum sexual function. Obstet Gynecol. 2012;19:647-55.

2. American Psychiatric Association. Diagnostic and Statistical Manual of Mental Disorders (DSM-5).

3. Abdool Z, Thakar R, Sultan AH. Postpartum female sexual function. Eur J Obstet Gynecol Reprod Biol. 2009;145:133-7.

4. Schwertner-Tiepelmann N, Thakar R, Sultan AH, Tunn R. Obstetric levator ani muscle injuries: current status. Ultrasound Obstet Gynecol. 2012;39:372-83.

5. Dietz HP, Lanzarone V. Levator trauma after vaginal delivery. Obstet Gynecol. 2005;106:707-12.

6. Dietz HP, Shek C, De Leon J, Steensma AB. Ballooning of the levator hiatus. Ultrasound Obstet Gynecol. 2008;31:676-80.

7. Durnea CM, O'Reilly BA, Khashan AS, et al. Status of the pelvic floor in young primiparous women. Ultrasound Obstet Gynecol. 2015;46:356-62.

8. van Delft K, Thakar R, Sultan AH, Schwertner-Tiepelmann N, Kluivers K. Levator ani muscle avulsion during childbirth: a risk prediction model. BJOG. 2014;121:1155-63. 
9. DeLancey JO, Morgan DM, Fenner DE, et al. Comparison of levator ani muscle defects and function in women with and without pelvic organ prolapse. Obstet Gynecol. 2007;109:295-302.

10. Lowenstein L, Gruenwald I, Gartman I, Vardi Y. Can stronger pelvic muscle floor improve sexual function? Int Urogynecol J. 2010;21:553-6.

11. Kanter G, Rogers RG, Pauls RN, Kammerer-Doak D, Thakar R. A strong pelvic floor is associated with higher rates of sexual activity in women with pelvic floor disorders. Int Urogynecol J. 2015;26: 991-6.

12. Martinez CS, Ferreira FV, Castro AAM, Gomide LB. Women with greater pelvic floor muscle strength have better sexual function. Acta Obstet Gynecol Scand. 2014;93:497-502.

13. van Delft K, Sultan AH, Thakar R, Schwertner-Tiepelmann N, Kluivers K. The relationship between postpartum levator ani muscle avulsion and signs and symptoms of pelvic floor dysfunction. BJOG. 2014;121:1164-71.

14. Thibault-Gagnon S, Yusuf S, Langer S, et al. Do women notice the impact of childbirth-related levator trauma on pelvic floor and sexual function? Results of an observational ultrasound study. Int Urogynecol J. 2014;25:1389-98.

15. van Delft KW, Thakar R, Sultan AH, IntHout J, Kluivers KB. The natural history of levator avulsion one year following childbirth: a prospective study. BJOG. 2015;122:1266-73.

16. Thibault-Gagnon S, McLean L, Goldfinger C, Pukall C, Chamberlain S. Differences in the biometry of the levator hiatus at rest, during contraction, and during Valsalva maneuver between women with and without provoked vestibulodynia assessed by transperineal ultrasound imaging. J Sex Med. 2016;13:243-52.

17. Speksnijder L, Oom DMJ, Van Bavel J, Steegers EAP, Steensma $\mathrm{AB}$. Association of levator injury and urogynecological complaints in women after their first vaginal birth with and without mediolateral episiotomy. Am J Obstet Gynecol. 2019;220:93.e1-9.

18. t Hoen LA, Utomo E, Steensma AB, Blok BF, Korfage IJ. The pelvic organ prolapse/urinary incontinence sexual questionnaire (PISQ-12): validation of the Dutch version. Int Urogynecol J. 2015;26:1293-303.
19. Dietz HP. Ultrasound imaging of the pelvic floor. Part II: threedimensional or volume imaging. Ultrasound Obstet Gynecol. 2004;23:615-25.

20. Serati M, Salvatore S, Siesto G, et al. Female sexual function during pregnancy and after childbirth. J Sex Med. 2010;7:2782-90.

21. Gałązka I, Drosdzol-Cop A, Naworska B, Czajkowska M, Skrzypulec-Plinta V. Changes in the sexual function during pregnancy. J Sex Med. 2015;12:445-54.

22. Kedde H, Donker G, Leusink P, Kruijer H. The incidence of sexual dysfunction in patients attending Dutch general practitioners. Int J Sex Health. 2011;23:269-77.

23. Aydın S, Bakar RZ, Aydın CA, Ateș S. Correlation between transperineal 3-dimensional ultrasound measurements of levator hiatus and female sexual function. Female Pelvic Med Reconstr Surg. 2017;23:433-7.

24. Abbott JA, Jarvis SK, Lyons SD, Thomson A, Vancaille TG. Botulinum toxin type a for chronic pain and pelvic floor spasm in women: a randomized controlled trial. Obstet Gynecol. 2006;108: 915-23.

25. Jarvis SK, Abbott JA, Lenart MB, Steensma AB, Vancaillie TG. Pilot study of botulinum toxin type a in the treatment of chronic pelvic pain associated with spasm of the levator ani muscles. Aust N Z J Obstet Gynaecol. 2004;44:46-50.

26. Angles-Acedo S, Ros-Cerro C, Escura-Sancho S, Elias-SabtoDomingo N, Palau-Pascual MJ, Espuna-Pons M. Coital resumption after delivery among OASIS patients: differences between instrumental and spontaneous delivery. BMC Womens Health. 2019;19: 154.

27. Gommensen D, Nohr E, Qvist N, Rasch V. Obstetric perineal tears, sexual function and dyspareunia among primiparous women 12 months postpartum: a prospective cohort study. BMJ Open. 2019;9:e032368.

Publisher's note Springer Nature remains neutral with regard to jurisdictional claims in published maps and institutional affiliations. 